\section{Radiology - Changing Role in Healthcare}

Dr. Md. Khalilur Rahman

MBBS, MPhil, MD

Associate Professor

Department of Radiology and Imaging

Dhaka Medical College, Dhaka, Bangladesh

In November 1895, X-rays were inadvertently discovered by the German physicist Wilhelm Conrad Roentgen. Roentgen provisionally named the new rays as X-rays using the mathematical label for something unidentified. Roentgen's initial manuscript, "On A New Kind of Rays" (áber eine neue Art von Strahlen), was published two months later and in 1901, he received the first ever Nobel Prize in Physics "in recognition of the extraordinary services he has rendered by the discovery of the remarkable rays" subsequently named after him. He himself refused to take out patents, intending to the free use of X-rays for the benefit of mankind. Today, Wilhelm Conrad Roentgen is honoured as the father of diagnostic radiology.1,2

Since the inadvertent discovery of X-rays, conventional radiography has developed greatly and mostly has been replaced by digital radiography equipments which convert X-ray images to electronic data that can be studied using a monitor and archived on a computer disk. Digital techniques permit the radiographs to be viewed instantaneously, additionally allow specific areas of the image to be enlarged, and the contrast of the images can be manipulated to provide greater visibility of the abnormality. ${ }^{1}$

The anatomical details and sensitivity of the newer modalities is now of a high order and the use of imaging for ultrastructural diagnostics nanotechnology, functional and quantitative diagnostics and molecular medicine is steadily increasing. Most recently the improved imaging clarity and tissue differentiation in a number of clinical situations has dramatically increased the spectrum of the diagnostic information and even in many cases revealing the pathology without the requirement of invasive tissue sampling. ${ }^{3}$
Eventually radiology is now the prime diagnostic aid for many diseases and also has a vital role in monitoring treatment and predicting outcome. Recent advancements in this field bring a number of imaging modalities which have differing physical principles of varying complexity. ${ }^{3}$

Accurate and timely diagnosis has always been the cornerstone of medical care. In the vast majority of conditions this involves clinical radiology, from the relatively simple chest $\mathrm{X}$-ray to diagnose pneumonia to the complexities of computed tomography (CT), magnetic resonance imaging (MRI) or positron emission tomography (PET) in the management of cancer. This diagnosis relies on the combination of imaging technology and the medical and diagnostic skills of the clinical radiologists making the diagnosis from the images. 4

The value of different modalities varies by disease and clinical situation and some modalities have certain limitations in some organ systems. The discipline of musculoskeletal radiology has evolved into a major imaging subspecialty in the years since the first use of $\mathrm{X}$-rays to diagnose fractures. Musculoskeletal radiology expertise has experienced enormous developments in diagnostic sensitivity and specificity and in image guided treatment options, in addition to technologic advances far beyond X-rays through advances in cross-sectional imaging such as CT and MR imaging. These two modern radiographic tools also play predominant role in neuroradiography. 5

The field of radiation medicine and nuclear imaging are both progressing rapidly with respect to technologic sophistication and multiplatform interface capabilities. Though PET is not really a new field, it has recently 
undergone a dramatic revitalization as new clinical indicators are validated for this type of functional imaging. PET recently has an immense positive role in the diagnosis and monitoring of cancer patients. PET-CT is now an indispensable tool in the detection of cancer and cancer therapy. 6

Most of us are familiar with the concept of diagnostic radiology in the field of medicine. However, numerous advancements in research have led radiology to play an increasingly promising part in health care not in just terms of spotting problems, but also as a major contributor to treatment and recovery. Working in tandem with other disciplines, radiology has had a major impact on achievements in such significant areas as early cancer detection, speedy trauma analysis, precise stroke localization and many others. 7

Interventional radiology, one of the subspecialty areas of radiology, uses the imaging modalities of diagnostic radiology to guide minimally invasive surgical procedures, such as that in laparoscopic gallstone surgery. The number of interventional radiology examinations has shown a huge rise, increasing by over $50 \%$ since 2007. Therapeutic radiology, better known as radiation oncology uses radiation to treat diseases such as cancer using a form of treatment called radiation therapy. 5,8

The last few decades have witnessed dramatic innovations and improvisations in imaging technology. In all sense radiological advances have revolutionized the practice of modern medicine. Imaging now uses a wide range of modalities that vary in their mode of image acquisition. In order to request the correct imaging technique and thereby improve patient management, it is useful for the practicing clinician to be conversant with all imaging techniques available, their advantages as well as limitations, indications and contraindications of each modality.

Delta Med Col J. Jan 2014;2(1):1-2

\section{References}

1. Sanghvi D, Harisinghani M G. Modalities in Modern Radiology: A Synopsis. J Postgrad Med. 2010;56:85-7.

2. Nobelprize.org [Internet]. The Nobel Prize in Physics 1901. Nobel Media AB 2013. [cited 2013 Dec 15]. Available from: http://www.nobelprize.org/nobel_prizes/ physics/ laureates/1901/.

3. European Society of Radiology 2009. The Future Role of Radiology in Healthcare. Insights Imaging. 2010;1(1):2-11.

4. Royal College of Radiology [Internet]. Investing in the Clinical Radiology Workforce - The Quality and Efficiency Case. [cited 2013 Dec 10]. Available from: http://www.rcr.ac.uk/docs/radiology/pdf/RCR_CRWorkf orce_June2012.pdf.

5. Sofka CM, Pavlov H. The history of clinical musculoskeletal radiology. Radiol Clin North Am. 2009;47(3):349-56.

6. Sharma N, Neumann D, Macklis R. The Impact of Functional Imaging on Radiation Medicine. Radiat Oncol [Internet]. 2008 [cited 2013 Dec 16];3:25. Available from: http://www.ro-journal.com/content/3/1/ 25.

7. Celebrate the Power of Imaging: the European Day of Radiology [Internet]. [cited 2013 Dec 15]. Available from:http://www.myesr.org/html/img/pool/EDoR_ 2011_General_Press_Release.pdf.

8. Dublin BC. Radiology Plays Big Role in Healthcare Delivery. Arab Times Kuwait English Daily [Internet]. Updated 2013 Dec 16 [cited 2013 Dec 16]. Available from: http://www.arabtimesonline.com/NewsDetails/ tabid/96/smid/414/ArticleID/151570/t/Radiologyplays-big- role-in-healthcare-delivery/Default.aspx. 